\title{
Evaluation of the health promotion standards in governmental and non-governmental hospitals in East-Azerbaijan
}

\author{
Mohammad Zakaria Pezeshki ${ }^{1}$, Mahasti Alizadeh ${ }^{1}$, Akbar Nikpajouh $^{2}$, Ali Ebadi $^{3}$, Soheila Nohi $^{3}$, Maryam Soleimanpour*1
}

Received: 18 Dec 2019

Published: 21 Oct 2019

\section{Abstract}

Background: Considering the importance of assessing the program of health promotion hospitals (HPH) for elucidating the compliance with the standards, the present study aimed to evaluate the health promotion standards in governmental and nongovernmental hospitals of East-Azerbaijan.

Methods: In the present cross-sectional study, all hospitals in East-Azerbaijan province in 2018 were recruited. The Persian validated World Health Organization (WHO) a self-assessment questionnaire was sent to the director of each hospital and invited to corporate with the study. Self-assessment questionnaire consists of 40 measurable elements that assess management policy, patient's assessment, patient information and intervention, promoting health work placed and continuity and cooperation. Independent sample ttest was conducted to compare the mean score of each standard across hospitals type, location, and size. A significance level of 0.05 was used.

Results: Hospitals total HPH score was $56.06 \pm 21.27$ (out of 100). Among five standards, Standard 3 had the highest score $(66.85 \pm 18.80)$, and Standard 4 had the lowest score $(47.79 \pm 19.12)$. The capital cities' hospitals had a significantly higher score in Standard $5(\mathrm{p}=0.02)$. Non-governmental hospitals had a significantly higher score in standard $4(\mathrm{p}=0.02)$. There were no significant differences in all five standards of HPH between hospitals with $\leq 200$ and $>200$ beds $(p>0.05)$.

Conclusion: The hospitals in East-Azerbaijan-Iran had moderate compliance with HPH program, and they need to improve their performance especially in the field of providing healthy workplace and offering proper education and health-promoting services to patients after discharge.

Keywords: Health promotion standards, Hospital, East Azerbaijan

Conflicts of Interest: None declared

Funding: Social Determinants of Health Research Center, Health Management, and Safety Promotion Research Institute, Tabriz University of Medical Sciences

\section{*This work has been published under CC BY-NC-SA 1.0 license.}

Copyright $($ Iran University of Medical Sciences

Cite this article as: Pezeshki MZ, Alizadeh M, Nikpajouh A, Ebadi A, Nohi S, Soleimanpour M. Evaluation of the health promotion standards in governmental and non-governmental hospitals in East-Azerbaijan. Med J Islam Repub Iran. 2019 (21 Oct);33:113. https://doi.org/10.47176/mjiri.33.113

\section{Introduction}

As defined by the world health organization, health is the "state of complete physical, social, and mental wellbeing and not just the absence of disease or infirmity" (1). In this regard, any attempt to promote these aspects of health education, disease prevention, and rehabilitation is

Corresponding author: Dr Maryam Soleimanpour, maryamsoleimanpour@gmail.com

1. Social Determinants of Health Research Center, Health Management and Safety Promotion Research Institute, Tabriz University of Medical Sciences, Tabriz, Iran

2. Rajaei Cardiovascular Medical and Research Center, Tehran, Iran

3. Deputy of Treatment, Tabriz University of Medical Sciences, Tabriz, Iran considered as health promotion. Different settings, including schools, workplaces, residential areas, and hospitals could have a role in health promotion (1).

Since hospitals are the principal to the health care system and spend more than $40 \%$ of health care expenditure,

$\uparrow$ What is "already known" in this topic:

HPH project had been started about three decades ago in Europe with the aim for decreasing in expenditures and implementing effective preventive programs in hospital, however, this project in Iran is new.

\section{$\rightarrow$ What this article adds:}

The hospitals in East-Azerbaijan-Iran had moderate compliance with HPH program and they need to improve their performance especially in the field of providing healthy workplace and offering proper education and health promoting services to patients after discharge. 
they are one of the major settings for the promotion of health and prevention of diseases beyond its traditional curative and diagnostic services (2). So, in this regard, in 1988 the WHO start the Health Promoting Hospitals $(\mathrm{HPH})$ project in Europe with the aim of decreasing expenditures and implementing effective preventive programs (3). The HPH emphasize on meeting the physical, mental and social needs of the patients, staffs, organization, and community and WHO established five standards of HPH including management policy, patient's assessment, patient information and intervention, promoting healthier workplace and continuity and cooperation (4). Health-promoting services are used in more than 900 hospitals worldwide, but most of these hospitals located in developed countries (5). The experience of developed countries has shown that the health-promotion program resulted in a reduction in costs and increasing the patients and staff's quality of life (6). Recently this program has been implemented in developing countries, and evaluation of this program in Taiwan showed that developing HPH programs in 52 hospitals in Taiwan resulted in positive effects on different aspects of hospitals, patients and staffs (7). This concept is new in Iran, and there is not much research to evaluate its effectiveness in Iranian hospitals. In a study in Isfahan, assessment of nine educational hospitals showed that based on health promotion score, only one hospital was at the good level (8). In another study in the northwest of Iran, form the administrative and clinical staff point of view, the "management policy" had the lowest score and "patient information and intervention" had the highest score in Tabriz heart hospital. The average score of compliance with the HPH standards $(1.60 \pm 0.40)$ indicated the moderate progress of this hospital towards the HPH standards (9).

Although the HPH project had been started about three decades ago in Europe with the aim of decreasing expenditures and implementing effective preventive programs in the hospital, this project in Iran is new. So, in its early stages, the assessment of its compliance with the WHO standards and also the determination of the barriers to program implementation would be useful for increasing the qualities of the services in hospitals. In this regard, this study aimed to evaluate the health promotion standards in governmental and non-governmental hospitals of EastAzerbaijan.

\section{Methods}

In the present cross-sectional study, the census method was used to recruit all hospitals in East-Azerbaijan province (28 hospitals in the capital city and 16 hospitals in suburban areas) in 2018. The baseline characteristics of hospitals including the type, size, and location were gathered from vice-chancellor for treatment in Tabriz University of medical sciences.

\section{Procedures}

The official invitation letter, including the aims of the study and also the explanation about HPH standards and a WHO self-assessment tool, was sent to the director of each hospital and invited to cooperate with the study.
They were asked to complete the questionnaire by a team of educational supervisor and accreditation manager.

Data collection was done using two questionnaires. The first one, including the questions regarding the relevant information about hospitals. The second questionnaire, WHO health-promoting hospital program self-assessment tool, is consists of 40 measurable elements ${ }^{10}$ that assess different domains related to HPH program, including management policy (nine elements), patient's assessment (seven elements), patient information and intervention (six elements), promoting a healthy workplace (ten elements) and finally continuity and cooperation (eight elements). Measurable elements are evaluated as 'yes, partly or no'. The total score for each standard was calculated by summing up the scores of each measurable elements. Considering that each standard consist of different number of elements, the score range ( 0 to 100 ) was converted by the following formula: $100 *$ total score for each standard/ maximum obtainable score.

The internal validity of the Persian version of the questionnaire was approved by the ministry of health and medical education (11).

\section{Statistical analysis}

The general characteristics of hospitals are presented as frequency distribution (number and \%). The HPH total score and score of each standard was presented as mean and standard deviation (mean $\pm \mathrm{SD}$ ). The KolmogorovSmirnov test was used for assessing normality of distribution. Independent sample t-test was conducted to compare the mean score of each standard across hospitals type (governmental versus non-governmental), location (capital city versus suburban areas) and size $(<200$ beds versus $>200$ beds). A significance level of 0.05 was used for all tests. SPSS18 was used for all statistical analyses.

\section{Results}

Table 1 presents the characteristics of included hospitals. About $63.6 \%$ of included hospitals were governmental, and $81.8 \%$ of them had less than 200 beds. About $63.6 \%$ of hospitals were located in the capital city.

Table 2 shows the mean of total score and measurable elements scores of health promotion standards in the East Azerbaijan hospitals. As can be seen, hospitals' total score was $56.06 \pm 21.27$ (out of 100). Between five standards, Standard 3 (Patient information \& intervention) had the highest score $(66.85 \pm 18.80)$, and Standard 4 (Promoting a healthy workplace) had the lowest score $(47.79 \pm 19.12)$.

The comparison of the health promotion standards score, according to hospitals' characteristics are presented

\begin{tabular}{|c|c|c|}
\hline Variables & Number & $\%$ \\
\hline \multicolumn{3}{|c|}{ Hospitals type } \\
\hline Governmental & 28 & 63.6 \\
\hline Non-governmental & 16 & 36.4 \\
\hline \multicolumn{3}{|l|}{ Size } \\
\hline$\leq 200$ beds & 36 & 81.8 \\
\hline$>200$ beds & 8 & 18.2 \\
\hline \multicolumn{3}{|l|}{ Hospital location } \\
\hline Capital city & 28 & 63.6 \\
\hline Suburban areas & 16 & 36.4 \\
\hline
\end{tabular}


Standard 1: Management policy (total score)

Standard 1 subscores

S1.1.The hospital's stated aims and mission include health promotion

$1.5 \pm 0.62$

$\mathrm{S} 1.2$. Minutes of the governing body reaffirm agreement within the past year to participate in the WHO HPH project

S1.3. The hospital's current quality and business plans include health promotion (HP) for patients, staff and the community

S1.4.The hospital identifies personnel and functions for the coordination of HP

S1.5.There is an identifiable budget for HP services and materials

S1.6.Operational procedures such as clinical practice guidelines or pathways incorporating HP actions are available in

S1.7. Specific structures and facilities required for health promotion (including resources, space, equipment) can be

S1.8. Data are routinely captured on HP interventions and available to staff for evaluation

S1.9. A program for quality assessment of the health-promoting activities is established

$0.88 \pm 0.81$

Standard 2: patient assessment (total score)

$0.88 \pm 0.81$
$55.10 \pm 20.88$

Standard 2 subscores

S 2.1. Guidelines on how to identify smoking status, alcohol consumption, nutritional status, psycho-social-economic status are present

$\mathrm{S}$ 2.2. Guidelines/procedures have been revised within the last year

$\mathrm{S}$ 2.3. Guidelines are present on how to identify needs for HP for groups of patients (e.g. asthma patients, diabetes patients, chronic obstructive pulmonary disease, surgery, rehabilitation)

$\mathrm{S}$ 2.4. The assessment is documented in the patient's record at admission

$\mathrm{S}$ 2.5. There are guidelines / procedures for reassessing needs at discharge or end of a given intervention

$\mathrm{S}$ 2.6. Information from referring physician or other relevant sources is available in the patient's record

$1.04 \pm 0.80$

$\mathrm{S}$ 2.7. The patient's record documents social and cultural background as appropriate

$0.81 \pm 0.81$

$0.97 \pm 0.73$

$1.30 \pm 0.74$

$1.00 \pm 0.71$

$1.27 \pm 0.76$

$1.25 \pm 0.69$

$66.85 \pm 18.80$

Standard 3 subscores

$\mathrm{S}$ 3.1. Information given to the patient is recorded in the patient's record

$1.29 \pm 0.70$

$1.06 \pm 0.69$

$\mathrm{S}$ 3.2. Health promotion activities and expected results are documented and evaluated in the records

S 3.3. Patient satisfaction assessment of the information given is performed and the results are integrated into the quali-

$1.40 \pm 0.69$

ty management system

S 3.4. General health information is available

$\mathrm{S}$ 3.5. Detailed information about high/risk diseases is available

$1.63 \pm 0.57$

$1.52 \pm 0.54$

$1.09 \pm 0.64$

$\mathrm{S}$ 3.6. Information is available on patient organizations

Standard 4: Promoting a healthy workplace (total score)

$1.09 \pm 0.64$
$47.79 \pm 19.12$

Standard 4 subscores

S 4.1. Working conditions comply with national/regional directives and indicators

$\mathrm{S}$ 4.2. Staff comply with health and safety requirements and all workplace risks are identified

S. 4.3. New staff receive an induction training that addresses the hospital's health promotion policy

S 4.4. Staff in all departments are aware of the content of the organization's health promotion policy

S.4.5 A performance appraisal system and continuing professional development including health promotion exists

$\mathrm{S}$ 4.6. Working practices (procedures and guidelines) are developed by multidisciplinary teams

$\mathrm{S}$ 4.7. Staff are involved in hospital policy-making, audit and review

S.4.8. Policies for awareness on health issues are available for staff

$1.09 \pm 0.64$

$1.23 \pm 0.64$

$1.20 \pm 0.66$

$1.00 \pm 0.68$

$1.13 \pm 0.72$

$0.81 \pm 0.62$

$0.79 \pm 0.66$

$1.04 \pm 0.68$

$0.56 \pm 0.69$

$0.72 \pm 0.75$

S.4.10. Annual staff surveys are carried out including an assessment of individual behavior, knowledge on supportive

services/policies, and use of supportive seminars

Standard 5: Continuity \& cooperation total score

Standard 5 subscores

S.5.1. The management board is taking into account the regional health policy plan

$54.10 \pm 21.85$

S.5.2. The management board can provide a list of health and social care providers working in partnership with the hospital

S.5.3. The intra- and intersectoral collaboration with others is based on the execution of the regional health policy plan

S.5.4. There is a written plan for collaboration with partners to improve the patients' continuity of care

S.5.5.Patients (and their families as appropriate) are given understandable follow-up instructions at out-patient consultation, referral or discharge

S.5.6.There is an agreed upon procedure for information exchange practices between organizations for all relevant patient information

S.6.7.The receiving organization is given in timely manner a written summary of the patient's condition and health needs, and interventions provided by the referring organization

If appropriate, a plan for rehabilitation describing the role of the organization and the cooperating partners is documented in the patient's record

*The standards are listed according to WHO manual and self-assessment form for health promotion hospitals (Reference 13).

in Table 3. As can be observed, the capital cities and nongovernmental hospitals had a higher score in all five standards compared with those of suburban areas hospitals. However, in terms of location of hospitals, the differences were only statistically significant in the case of
Standard $5(\mathrm{p}=0.02)$ and in terms of the type of hospitals, the difference was only significant in standard $4(\mathrm{p}=0.02)$. There were no significant differences in all five standards of HPH between hospitals with $\leq 200$ and $>200$ beds $(\mathrm{p}>0.05)$. 


\begin{tabular}{|c|c|c|c|c|c|}
\hline Variables & Standard 1 & Standard 2 & Standard 3 & Standard 4 & Standard 5 \\
\hline \multicolumn{6}{|l|}{ Hospital Location } \\
\hline Capital city hospitals & $58.07 \pm 21.15$ & $57.93 \pm 19.84$ & $69.94 \pm 17.02$ & $49.10 \pm 15.98$ & $60.51 \pm 16.18$ \\
\hline Suburban areas hospitals & $47.03 \pm 26.04$ & $50.00 \pm 22.42$ & $61.45 \pm 21.05$ & $45.33 \pm 24.38$ & $43.26 \pm 26.32$ \\
\hline p-value* & 0.12 & 0.24 & 0.15 & 0.54 & 0.02 \\
\hline \multicolumn{6}{|l|}{ Hospital type } \\
\hline Governmental & $53.24 \pm 15.06$ & $50.64 \pm 20.06$ & $66.66 \pm 18.46$ & $41.25 \pm 13.50$ & $57.38 \pm 16.96$ \\
\hline Non-governmental & $63.49 \pm 24.81$ & $62.94 \pm 18.66$ & $72.39 \pm 16.20$ & $55.00 \pm 15.49$ & $63.63 \pm 15.51$ \\
\hline p-value* & 0.22 & 0.11 & 0.38 & 0.02 & 0.37 \\
\hline \multicolumn{6}{|l|}{ Number of beds } \\
\hline$<200$ & $55.38 \pm 24.98$ & $55.51 \pm 20.20$ & $67.12 \pm 18.78$ & $49.28 \pm 20.00$ & $53.87 \pm 23.29$ \\
\hline$>200$ & $50.69 \pm 16.38$ & $53.06 \pm 25.70$ & $65.62 \pm 20.13$ & $55.20 \pm 14.47$ & $55.20 \pm 14.47$ \\
\hline $\mathrm{p}^{*}$ & 0.61 & 0.78 & 0.84 & 0.28 & 0.89 \\
\hline
\end{tabular}

\section{Discussion}

In the present study, the health promotion standards in governmental and non-governmental hospitals of EastAzerbaijan was studied. According to the results of this study, the total HPH score of East-Azerbaijan hospitals was $56.06 \pm 21.27$ that is higher than those of educational hospitals in Isfahan (48.80 \pm 9.80$)(8)$ and also 38 hospitals from four provinces of Iran $(54.1 \pm 15.1)(12)$. These finding indicated that although hospitals in East-Azerbaijan had better compliance with HPH program compared with those of other provinces of Iran, they had a long distance to accomplish the objectives of HPH standards. Hospitals in other developing and developed countries had a better score in HPH standards $(7,13)$. These differences may be due to this fact that in Iran hospitals are more treatmentoriented and had little role in educating and promotion of healthy life style (14).

In line with previous studies conducted in $\operatorname{Iran}(8,12)$, in the present study, standard three (Patient information \& intervention) had the highest score $(66.85 \pm 18.80)$ in both governmental and non-governmental hospitals; it indicated that the appropriate information about the patients' disease and all information that may affect their health have been provided.

East Azerbaijan hospitals had the lowest score $(47.79 \pm 19.12)$ in standard 4 (Promoting a healthy workplace). In a study in Hamadan, Hamidi et al. also reported the lowest score for standard 4 among the all HPH standards (14). This showed that although hospitals had a proper function in promoting health-related problems of patients, its role in promoting a healthy place for their staff was not appropriate. Considering that hospital staffs are one of the most endangered working population and their health are directly related to their function and consequently patient's health, our hospitals should have a proper plan for promoting this standard. Although nongovernmental hospitals had significantly better function compared with governmental hospitals, the mean score of these hospitals was still low. Moreover, we observed that the lowest score in this standard was related to two subscales "smoking cessation programs are offered"and "staff is involved in hospital policy-making, audit, and review". It is obvious that in our hospitals, the personnel had no active role in hospital decision making. So this may affect their implementation. Additionally, not offering healthy behavior to personnel such as smoking cessation programs showed that the knowledge and awareness of the staff in our hospitals about the significance of the health promotion programs are disregarded. So for having more compliance with HPH programs in our hospitals, staff education and also empowerment should be emphasized (14).

In the present study, we showed that the capital city hospitals had the highest scores in all five standards compared with those of suburban area hospitals. However, these differences were only statistically significant in the case of Standard 5 (Continuity \& cooperation). Among the five standards, the suburban area hospitals had the lowest score in standard 5. It indicated that although these hospitals had an important role in the patient's treatment. However, they had no proper control on the health-promoting lifestyle of patients after they discharged.

In the present study, all hospitals of Tabriz were included to assess the compliance with WHO HPH program. However, the important limitation of the present study is that the results were based on the self-assessment report of the hospitals that may prone to bias.

\section{Conclusion}

In conclusion, considering the results, the hospitals in East-Azerbaijan-Iran had moderate compliance with HPH program, and they need to improve their performance especially in the field of providing healthy workplace and offering proper education and health-promoting services to patients after discharge.

\section{Acknowledgments}

The authors wish to thanks social determinants of health research center, Health management, and safety promotion research institute, Tabriz University of Medical Sciences for financial support. The results of the present study are derived from the Maryam Soleimanpour residency thesis in community medicine (Thesis NO: 58609).

\section{Conflict of Interests}

The authors declare that they have no competing interests.

\section{References}

1. Kumar S, Preetha G. Health promotion: an effective tool for global health. Indian J Commun Med. 2012;37(1):5-12.

2. Groene O, Jorgensen SJ. Health promotion in hospitals-a strategy to 
improve quality in health care. Eur J Public Health. 2005;15:6-8.

3. Lee CB, Chen MS, Powell MJ, Chu CMY. Organisational change to health promoting hospitals: a review of the literature. Springer Sci Rev. 2013;1:13-23.

4. Grone O. Developing standards for health promotion in hospitals. Results of a pilot test in nine European countries. Report on a WHO Workshop, Barcelona, Spain. WHO EURO, Barcelona, EUR/03/5038045; 2003.

5. Organization WH. The International Network of Health Promoting Hospitals and Health Services: integrating health promotion into hospitals and health services: concept, framework and organization. 2007.

6. Whitehead D. The European Health Promoting Hospitals (HPH) project: how far on? Health Promot Int. 2004;19:259-267.

7 Lin YW, Huang HL, Tung SC. The organisational diagnosis of a Health Promoting Hospital in Taiwan. Patient Educ Couns. 2009;76:248-253

8. Afshari A, Mostafavi F, Keshvari M, Ahmadi-Ghahnaviye L, Piruzi M, Moazam E, et al. Health promoting hospitals: a study on educational hospitals of Isfahan, Iran. Health Promot Perspect. 2016;6:23.

9. Taghdisi MH, Poortaghi S, Suri JV, Dehdari T, Gojazadeh M, Kheiri M. Self-assessment of health promoting Hospital's activities in the largest heart Hospital of Northwest Iran. BMC Health Serv Res. 2018;18:572.

10. Nikpajouh A. Standards of health promoting hospitals: 68 or 40 measurable elements? Health Promot Perspect. 2017;7:109.

11. Nikpajouh A, Shahrbaf MA, Doayie M, Mohseny M, Ebadi A, Alizadeh $\mathrm{M}$, et al. Health promoting hospitals in Iran: Persian translation, cultural adaptation, content and face validation of selfassessment form of the standards of health promoting hospitals affiliated to the World Health Organization. Med J Islam Repub Iran. 2018:32:120.

12. Yaghoubi M, Javadi M. Health promoting Hospitals in Iran: How it is. J Educ Health Promot. 2013;2.

13. Groene O, Alonso J, Klazinga N. Development and validation of the WHO self-assessment tool for health promotion in hospitals: results of a study in 38 hospitals in eight countries. Health Promot Int 2010;25:221-229.

14. Hamidi Y, Hazavehei SMM, Karimi-Shahanjarini A, SeifRabiei MA, Farhadian M, Alimohamadi S, et al. Investigation of health promotion status in specialized hospitals associated with Hamadan University of Medical Sciences: health-promoting hospitals. Hosp Pract. 2017:45:215-221 